\title{
CARACTERÍSTICAS DOS REPOSITÓRIOS DE DADOS CIENTÍFICOS NO BRASIL
}

\author{
Lucas Nóbrega Paganine \\ Pós-graduado lato sensu em Gestão Pública pela \\ Faculdade Unyleya. Bolsista pesquisador do Programa de \\ Capacitação Institucional - modalidade \\ Desenvolvimento/categoria D (PCI-D/D) no Instituto \\ Brasileiro de Informação em Ciência e Tecnologia (IBICT). \\ Inpaganine@hotmail.com \\ https://orcid.org/0000-0001-8978-4742
}

Bianca Amaro

Doutora em Linguística Aplicada pela Universidade Pompeu Fabra (UPF) - Espanha. Coordenadora do Programa Brasileiro de Acesso Aberto no Instituto Brasileiro de Informação em Ciência e Tecnologia (IBICT). Ganhadora do prêmio internacional Electronic Publishing Trust for Development (EPT 2015).

bianca@ibict.br

https://orcid.org/0000-0002-4703-8992

\section{RESUMO}

O objetivo do estudo em questão é observar e descrever os repositórios de dados científicos no Brasil. Para tanto, foi realizada uma análise descritiva, iniciada a partir de um levantamento na plataforma "re3data". Esse levantamento resultou nos nove repositórios de dados científicos do Brasil, ali cadastrados. A análise foi orientada pela metodologia desenvolvida, adaptada a partir das características essenciais de um repositório científico. Estas foram adaptadas para a realidade dos dados de pesquisa e, então, organizadas em categorias, entre elas, - Armazenamento, a Descrição e a Apresentação dos itens. O baixo número de repositórios, a não padronização e a inconformidade com padrões internacionais estabelecidos são indicativos de que a realidade Brasileira ainda não está preparada, adequadamente, para lidar com as questões que vêm surgindo.

Palavras-chave: Repositório de dados. Dados de pesquisa. re3data.

\section{CHARACTERISTICS OF SCIENTIFIC DATA REPOSITORIES IN BRAZIL}

\begin{abstract}
The aim of the study visto observe and describe the repositories of scientific data in Brazil. For this purpose, a descriptive analysis was carried out, starting from a survey on the re3data platform. This survey resulted in the nine scientific data repositories in Brazil registered there. The analysis was guided by the developed methodology, adapted from the essential characteristics of a scientific repository. The characteristics were adapted to the reality of the research data and the organized into categories (Storage, Description and Presentation of the items). The low number of repositories, non-standardization and non-compliance with established international standards are indicative that the Brazilian reality is not yet adequately prepared to deal with the issues arising.
\end{abstract}

Keywords: Data repository. Research data. re3data.

Recebido em: 21/03/2020

Aceito em: 30/07/2020

Publicado em: 11/09/2020

\section{INTRODUÇÃO}

Com o surgimento das novas tecnologias da informação e comunicação (TIC), novas formas como as pessoas interagem e se comunicam, foram desencadeadas. Por consequência, essas mudanças têm gerado, também, significativos processos de transformação na comunicação científica. Nesse cenário de transformações, o movimento 
Open Access surgiu apresentando novas soluções, em contrapartida aos problemas desenvolvidos, devido ao sistema de mercado tradicional dos periódicos científicos comerciais.

Sarmento et al. (2005, p. 8) esclarece que "existem três declarações que regem o movimento Acesso Livre, ou seja, as declarações de Budapeste, Bethesda e Berlim. As três apoiam o acesso global e irrestrito ao conhecimento científico" e são descritas, em linhas gerais, por Costa e Leite (2015, p. 3) ao dizer que elas "reuniram iniciativas de sistemas de informação e procedimentos já existentes, que promoviam o acesso amplo às publicações científicas". Dessa forma, as declarações apresentaram orientações para o desenvolvimento de novos sistemas e ficaram conhecidos como fundadores do Movimento de Acesso Aberto.

Dentre as iniciativas que formalizam e orientam o movimento Open Access, destaca-se, aqui, a declaração de Bethesda, que evidenciou, ainda em 2003, a importância do acesso aberto para as ciências da saúde (SARMENTO; et al., 2005). A declaração de Bethesda formalizou a demanda para o tratamento dos dados de pesquisa, no contexto do acesso aberto.

Os repositórios de dados de pesquisa têm sido sistematicamente propostos pela literatura, como a ferramenta adequada para o compartilhamento desses dados de pesquisa. No entanto ainda se encontram diversos desafios, como a padronização dos sistemas e a curadoria dos dados. Ao se considerar o contexto apresentado e a relevância e atualidade do tema, este estudo busca identificar a situação atual de desenvolvimento dos repositórios de dados de pesquisa, no Brasil.

\section{METODOLOGIA}

Para execução da análise proposta, foi realizada uma pesquisa de levantamento e análise descritiva, que, segundo Gil (2011, p. 28) "têm como objetivo primordial a descrição das características de determinada população ou fenômeno ou o estabelecimento de relações entre variáveis", acerca dos repositórios de dados no Brasil, registrados no re3data. O levantamento dos dados ocorreu em março, do ano 2018.

A análise metodológica desenvolvida foi sintetizada e adaptada, a partir das três características essenciais para um repositório de dados, proposta por Rodrigues et. al. (2010). Essas características foram utilizadas como categorias de análise dos dados 
coletados. Portanto, os resultados foram sistematizados em três grandes conjuntos: armazenamento, descrição e apresentação dos itens, como ilustrado pelo quadro 2, a seguir.

Quadro 1- Categorias e itens analisados.

\begin{tabular}{|l|l|}
\hline \multicolumn{1}{|c|}{ Categorias } & \multicolumn{1}{c|}{ Elementos analisados } \\
\hline \multirow{2}{*}{ Armazenamento } & Software: programa(s) utilizado(s) no repositório. \\
\cline { 2 - 3 } & Sistema de preservação: métodos ou práticas de preservação utilizadas. \\
\cline { 2 - 2 } Descrição & Licença: tipo de licença(s) utilizada. \\
\hline \multirow{2}{*}{ Categorias } & Metadados (padrão): qual padrão de metadados utilizado. \\
\hline \multirow{2}{*}{ Apresentação dos itens } & \multicolumn{1}{|c|}{ Elementos analisados } \\
\hline & $\begin{array}{l}\text { Tipo de conteúdo: caracterização dos dados apresentados no } \\
\text { repositório, como por exemplo, dados audiovisuais, textuais, etc. }\end{array}$ \\
\cline { 2 - 3 } & $\begin{array}{l}\text { Quantidade de itens: número de entradas de itens no repositório. } \\
\text { com o todo. }\end{array}$ \\
\hline & $\begin{array}{l}\text { Relação do conjunto com a publicação: se há indicação da relação do } \\
\text { conjunto de dados com a publicação, onde se encontra.? }\end{array}$ \\
\hline
\end{tabular}

Fonte: Adaptado de Rodrigues et al. (2010).

As adaptações da metodologia foram realizadas com o objetivo de sintetizar as tabelas e as adaptar à realidade dos repositórios brasileiros. Nesse sentido, foram retirados os quesitos Repositório aberto e URL persistente da categoria Armazenamento. Por fim, os elementos "Relação do item com o conjunto" e "Relação do conjunto com a publicação" foram conjugados.

\section{RESULTADOS E DISCUSSÃO}

Partindo da metodologia estabelecida, foram recuperados 9 repositórios, entre as áreas: Ciências humanas e sociais, Geociências, Ciências da vida e Engenharias. Pode-se perceber que os temas estão distribuídos de forma homogênea, exceto entre as Engenharias, em que aparece em apenas um repositório. 
Quadro 2 - Repositórios de dados de pesquisa no Brasil

\begin{tabular}{|c|c|c|c|}
\hline Nome do repositório & Área & $\begin{array}{l}\text { Acesso } \\
\text { aberto? }\end{array}$ & Breve descrição \\
\hline $\begin{array}{l}\text { Brazil Exploration and Production } \\
\text { Database }\end{array}$ & Geociências & Sim & $\begin{array}{l}\text { Inaugurado em 2000, armazena, } \\
\text { organiza e disponibiliza informações } \\
\text { geofísicas, geológicas e geoquímicas, } \\
\text { além de processar e analisar. }\end{array}$ \\
\hline World Clim - Global Climate Data & Geociências & Sim & $\begin{array}{l}\text { Apresenta grades e conjuntos de } \\
\text { camadas climáticas para mapeamento } \\
\text { e modelagem espacial. }\end{array}$ \\
\hline GLOBE & $\begin{array}{l}\text { Ciências } \\
\text { da vida }\end{array}$ & Sim & $\begin{array}{l}\text { O Global Collaboration Engine é um } \\
\text { ambiente online colaborativo voltado } \\
\text { para variação ambiental, integrando } \\
\text { estudos locais e regionais a } \\
\text { dados globais }\end{array}$ \\
\hline IBICT Dataverse Network & $\begin{array}{l}\text { Ciências } \\
\text { Humanas } \\
\text { e Sociais }\end{array}$ & Sim & $\begin{array}{l}\text { Trata de preservação de longo prazo } \\
\text { e de boas práticas de arquivamento, } \\
\text { voltado para os participantes da } \\
\text { rede Cariniana. }\end{array}$ \\
\hline $\begin{array}{l}\text { International Ocean } \\
\text { Discovery Program }\end{array}$ & Geociências & Sim & $\begin{array}{l}\text { O International Ocean Discovery } \\
\text { Program (IODP) é uma colaboração } \\
\text { internacional de pesquisa marinha, } \\
\text { que recupera dados de sedimentos e } \\
\text { rochas do fundo do mar e monitora } \\
\text { ambientes subterrâneos. }\end{array}$ \\
\hline \multicolumn{4}{|c|}{ Conclusão } \\
\hline $\begin{array}{l}\text { Base de Dados Científicos da } \\
\text { Universidade Federal do Paraná }\end{array}$ & $\begin{array}{l}\text { Ciências } \\
\text { Humanas e } \\
\text { Sociais }\end{array}$ & Sim & $\begin{array}{l}\text { Reúne os dados científicos utilizados } \\
\text { nas pesquisas publicadas pela } \\
\text { comunidade da UFPR em teses, } \\
\text { dissertações, artigos de periódicos e } \\
\text { outros materiais bibliográficos }\end{array}$ \\
\hline PPBio Data Repository & $\begin{array}{c}\text { Ciências da } \\
\text { vida }\end{array}$ & Sim & $\begin{array}{l}\text { O Programa de Pesquisa em } \\
\text { Biodiversidade (PPBio) foi criado em } \\
2004 \text { integrando atividades de } \\
\text { pesquisa e disseminando resultados } \\
\text { em diversas finalidades, incluindo } \\
\text { gestão ambiental e educação. Seu } \\
\text { repositório trabalha com dados } \\
\text { ecológicos complexos. }\end{array}$ \\
\hline
\end{tabular}


Continuação

\begin{tabular}{|c|c|c|c|}
\hline Nome do repositório & Área & $\begin{array}{l}\text { Acesso } \\
\text { aberto? }\end{array}$ & Breve descrição \\
\hline \multicolumn{4}{|c|}{ Conclusão } \\
\hline $\begin{array}{l}\text { CEDAP Research Data } \\
\text { Repository - research data }\end{array}$ & Multidisciplinar & Sim & $\begin{array}{l}\text { O CEDAP da UFRGS objetiva reunir } \\
\text { os dados científicos utilizados na } \\
\text { pesquisa, nas diversas áreas do } \\
\text { conhecimento. O repositório visa } \\
\text { reunir os dados, com a } \\
\text { documentação, a fim de proporcionar } \\
\text { um ambiente de estudo das } \\
\text { metodologias, de uso e reutilização } \\
\text { dos dados. E mantida em parceria } \\
\text { com o CPD da UFRGS. }\end{array}$ \\
\hline Open Research Data @PUC-Rio & Engenharias & $\operatorname{Sim}$ & $\begin{array}{l}\text { É um agregador para facilitar o } \\
\text { acesso aos dados de nova pesquisa, } \\
\text { entre muitos outros conteúdos } \\
\text { digitais no Repositório Maxwell. }\end{array}$ \\
\hline
\end{tabular}

Fonte: Adaptado de RE3DATA.

Um fator relevante a ser destacado é que os únicos repositórios, exclusivamente, brasileiros são os Exploration and Production Data Bank, Base de Dados Científicos da Universidade Federal do Paraná, Open Research Data @PUC-Rio e o CEDAP Research Data Repository - research data. Configurando, apenas, pouco menos do que metade dos repositórios cadastrados. O IBICT Dataverse Network e PPBio Data Repository são repositórios que, de acordo com o RE3DATA, também estão inclusos na categoria internacional. O GLOBE é uma iniciativa em conjunto com os Estados Unidos, enquanto o IODP, é uma colaboração de diversos países.

\subsection{Armazenamento}

A categoria inicial de análise, proposta pela metodologia, descreve o armazenamento dos dados de pesquisa, a partir dos aspectos referentes a: a) software utilizado pelo repositório; b) existência de sistemas de preservação e c) tipos de licenças utilizadas de acesso ao conteúdo. Vale destacar que todos os repositórios estudados são de acesso aberto.

a) Software - As informações acerca dos softwares utilizados foram estruturadas no quadro 3, a seguir, permitindo-se perceber que, no momento, não há uma predominância de escolha ao se tratar do assunto. Isso inclui, até mesmo, 


\section{Biblos E-ISSN 2236-7594}

o desenvolvimento de programas próprios e específicos, como o caso do World Clim e do GLOBE.

Quadro 3 - Armazenamento: Software

\begin{tabular}{|l|l|}
\hline \multicolumn{1}{|c|}{ Nome do repositório } & \multicolumn{1}{c|}{ Nome do software } \\
\hline $\begin{array}{l}\text { Base de Dados Científicos da Universidade } \\
\text { Federal do Paraná }\end{array}$ & Dspace \\
\hline $\begin{array}{l}\text { CEDAP Research Data Repository - } \\
\text { research data }\end{array}$ & Metacat \\
\hline PPBio Data Repository & World Clim Version 2.0 \\
\hline World Clim - Global Climate Data & $\begin{array}{l}\text { ISEA3H Level 12 DGG, generated using } \\
\text { DGGRID software version 3.1 }\end{array}$ \\
\hline GLOBE & Data Verse \\
\hline IBICT Dataverse Network & Não informado \\
\hline International Ocean Discovery Program & Maxwell System (próprio) \\
\hline Exploration and Production Data Bank &
\end{tabular}

Fonte: Elaboração própria.

Dentre os softwares encontrados, o Dspace, o Metacat e o DataVerse merecem uma atenção especial. O Dspace é um software livre, voltado para a criação de repositórios, realizando armazenamento, gerenciamento, preservação e visibilidade. O Metacat é um repositório de dados flexível e de código aberto, voltado para dados da ecologia e ciências ambientais. E o Dataverse, também de código-fonte aberto, foi desenvolvido em Harvard para compartilhar, preservar, citar, explorar e analisar dados de pesquisa de uma forma geral. Cada repositório é uma instalação que, por sua vez, abriga diversos dataverses.

b) Sistemas de preservação - Com relação aos sistemas de preservação, os repositórios que informaram adotar algum tipo qualquer de medida, foram:

$>$ IBICT Dataverse Network com o Harvard Dataverse Preservation Policy, que estabelece termos e datas de backups e procedimentos para arquivamento digital.

> International Ocean Discovery Program, em seu ODP Sample Distribution, Data Distribution, and Publications Policy, que define a preservação 
como uma das obrigações do programa, visando o arquivamento para análises futuras. Ele apresenta, também, a forma como esse arquivamento era realizado, antes de 1997 e como é hoje, reiterando que a preservação é um dos requisitos para uma estratégia de sucesso de amostragem.

CEDAP Research Data Repository - research: determina a data que declara apenas oferecer serviço de repositório que inclui preservação digital; e

- Open Research Data @PUC-Rio que declara ser membro da Cooperativa Meta Archive (https://www.metaarchive.org/) e seus dados estão sendo preparados para serem incluídos no processo de preservação.

Vale destacar que, dentre os repositórios analisados, apenas a Base de Dados Científicos da Universidade Federal do Paraná e o CEDAP Research Data Repository não disponibilizam uma política de dados.

c) Licença - Percebe-se uma preferência pela licença Creative Commons (licenças que facilitam o compartilhamento e reuso de forma menos restringível), mais adequadas para ambientes de acesso aberto. Logo após, temos a Copyright (direito autoral que impede exploração, sem prévia autorização) combinada com outro fator, como por exemplo, licenciamento ou termos de uso.

Quadro 4 - Armazenamento: Licença

\begin{tabular}{|l|l|}
\hline \multicolumn{1}{|c|}{ Nome do repositório } & \multicolumn{1}{|c|}{ Licença utilizada } \\
\hline World Clim - Global Climate Data & \multirow{2}{*}{ Copyright \& Licensing } \\
\hline $\begin{array}{ll}\text { Base de Dados Científicos da Universidade } \\
\text { Federal do Paraná }\end{array}$ & \\
\hline CEDAP Research Data Repository & \\
\hline Open Research Data @PUC-Rio & \multirow{2}{*}{ Copyright \&Terms of use } \\
\hline PPBio Data Repository & \\
\hline Exploration and Production Data Bank & Public Domain \\
\hline IBICT Dataverse Network & CC BY \\
\hline GLOBE &
\end{tabular}

Fonte: Elaboração própria. 
Sobre a categoria armazenamento, percebe-se, então, que não há um software predominante utilizado. Assim, a preservação não recebe a preocupação devida e não existe consenso na escolha de licenças utilizadas ainda, levando-se em conta o fato de todos os repositórios se declararem como de acesso aberto, mesmo contando com dados, tanto abertos quanto restritos e embargados. Vale ressaltar, também, que os uploads de dados são todos restritos a instituições membros ou ao registro, com exceção dos World Clime Exploration and Production Data Bank, que estão fechados.

\subsection{Descrição}

A próxima categoria da análise abordou os padrões de metadados para a descrição dos conteúdos disponibilizados nos repositórios de dados de pesquisa estudados.

- Metadados - Os padrões de metadados encontrados foram: o Dublin Core, padrão criado para dados bibliográficos; EML para dados ecológicos; a ISO 19115, que orienta dados geográficos; e o DDI, que descreve dados de métodos observacionais de ciências sociais.

Quadro 5 - Descrição: Metadados

\begin{tabular}{|l|l|}
\hline \multicolumn{1}{|c|}{ Nome do repositório } & \multicolumn{1}{c|}{ Padrão de metadados } \\
\hline $\begin{array}{l}\text { Base de Dados Científicos da Universidade } \\
\text { Federal do Paraná }\end{array}$ & Dublin Core \\
\hline CEDAP Research Data Repository - research data & $\begin{array}{l}\text { EML - Ecological Metadata Language } \\
\text { (protocolo NetCDF) }\end{array}$ \\
\hline PPBio Data Repository & ISO 19115 (formato REST e protocolo OAI-PMH) \\
\hline International Ocean Discovery Program & $\begin{array}{l}\text { DDI - Data Documentation Initiative } \\
\text { (formato SWORD) }\end{array}$ \\
\hline IBICT Dataverse Network & Outro (próprio) \\
\hline GLOBE &
\end{tabular}

Fonte: Elaboração própria.

Não foi encontrado, então, qualquer predominância na escolha do padrão de metadados, com inclusive o repositório GLOBE, declarando utilizar um padrão próprio. Não foram encontradas informações a respeito dos metadados, utilizados pelos repositórios World Clim - Global Climate Data, Exploration and Production Data Bank e o Open Research Data@PUC-Rio. 


\subsection{Apresentação}

Por fim, a categoria "Apresentação" aborda informações sobre os tipos de recursos disponibilizados nos repositórios, como, por exemplo, volume de itens e suas relações.

- Tipo de conteúdo - Os tipos de conteúdo encontrados nos repositórios foram: imagens, documentos office padrão, gráficos estruturados, texto sem formatação, texto estruturado, dados científicos ou estatísticos, bases de dados, códigos-fonte, aplicações de software, dados arquivados, dados audiovisuais, dados brutos e dados baseados em redes.

Quadro 6 - Tipos de conteúdo

\begin{tabular}{|c|c|}
\hline Nome do Repositório & Tipos de conteúdo \\
\hline World Clim - Global Climate Data & $\begin{array}{l}\text { Gráficos estruturados, dados científicos e estatísticos, } \\
\text { outros e dados brutos }\end{array}$ \\
\hline International Ocean Discovery Program & $\begin{array}{l}\text { Documentos padrão do office, imagens, gráficos } \\
\text { estruturados, dados científicos e estatísticos, dados } \\
\text { brutos, texto sem formatação e texto estruturado }\end{array}$ \\
\hline GLOBE & $\begin{array}{l}\text { Documentos padrão do office, dados baseados em } \\
\text { rede, gráficos estruturados, dados brutos dados } \\
\text { arquivados e outros }\end{array}$ \\
\hline IBICT Dataverse Network & $\begin{array}{l}\text { Dados científicos e estatísticos, texto sem formatação, } \\
\text { outros e dados brutos }\end{array}$ \\
\hline PPBio Data Repository & $\begin{array}{l}\text { Documentos padrão do office, imagens, dados } \\
\text { audiovisuais, texto estruturado, texto sem formatação, } \\
\text { dados científicos e estatísticos e dados brutos }\end{array}$ \\
\hline $\begin{array}{l}\text { CEDAP Research Data Repository - } \\
\text { research data }\end{array}$ & $\begin{array}{l}\text { Documentos padrão do office, dados arquivados, texto } \\
\text { estruturado, texto sem formatação, dados científicos e } \\
\text { estatísticos, dados audiovisuais, imagens e bases } \\
\text { de dados }\end{array}$ \\
\hline \multicolumn{2}{|r|}{ Conclusão } \\
\hline $\begin{array}{l}\text { Base de Dados Científicos da Universidade } \\
\text { Federal do Paraná }\end{array}$ & $\begin{array}{l}\text { Texto sem formatação, dados audiovisuais, imagens, } \\
\text { dados arquivados, aplicativos de software, gráficos } \\
\text { estruturados, código fonte, dados científicos e } \\
\text { estatísticos e bases de dados }\end{array}$ \\
\hline Exploration and Production Data Bank & $\begin{array}{l}\text { Dados científicos e estatísticos, texto sem formatação, } \\
\text { gráficos estruturados documentos padrão do office } \\
\text { e imagens }\end{array}$ \\
\hline Open Research Data@PUC-Rio & $\begin{array}{l}\text { Outros dados científicos e estatísticos e documentos } \\
\text { padrão do office }\end{array}$ \\
\hline
\end{tabular}

Fonte: Elaboração própria. 


\section{Biblos E-ISSN 2236-7594}

Podemos, então, perceber uma predominância do tipo: dados científicos ou estatísticos. Os tipos que menos surgem são exatamente as tipificações mais específicas tecnológicas, ou seja, os códigos-fonte, as aplicações de software e os dados baseados em rede:

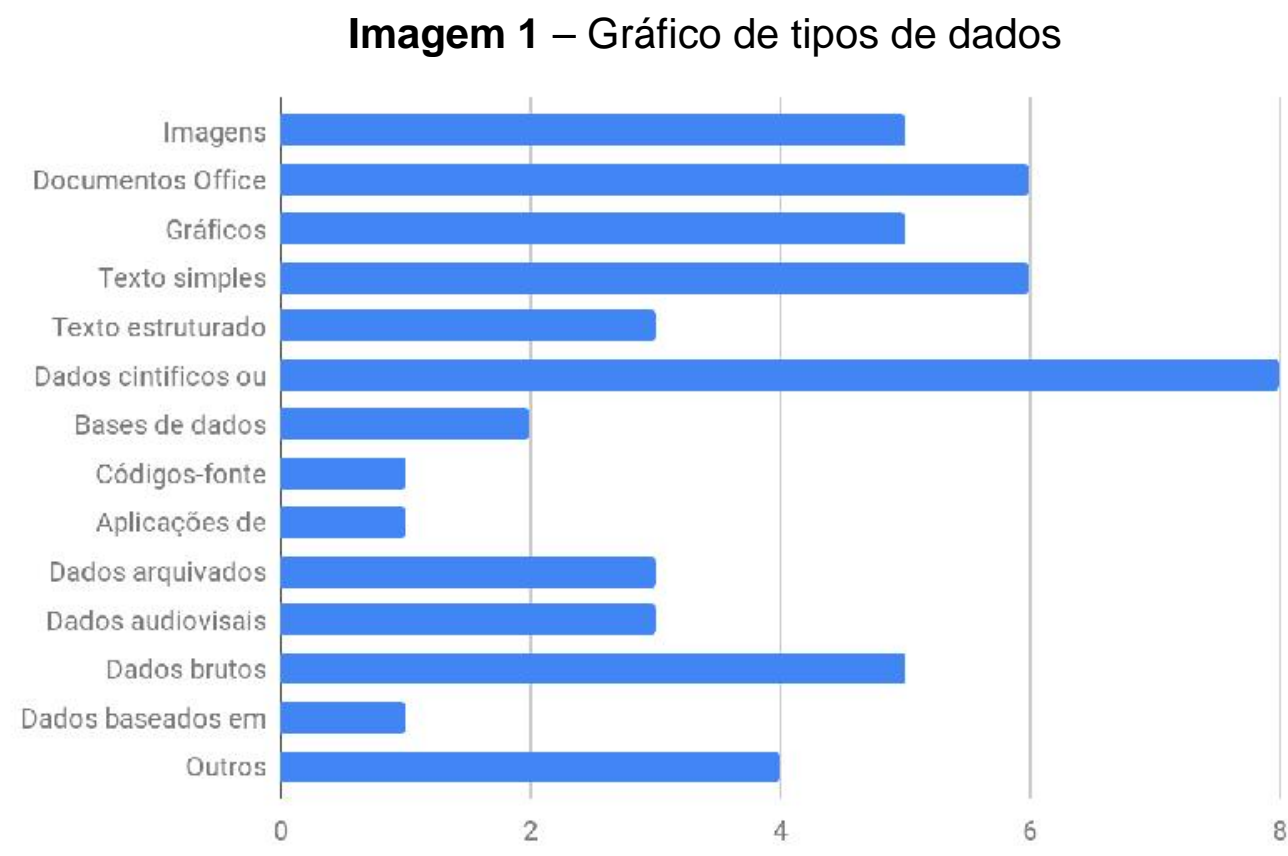

Fonte: Elaboração própria.

- Quantidade de itens - Relativo à quantidade de itens dos repositórios, não foram encontradas informações sobre o International Ocean Discovery Program. E sobre o Exploration and Production Data Bank, apenas é informado o volume digital, no caso 6 petabytes. Para os outros, foi elaborado o quadro a seguir:

Quadro 7-Quantidade de itens no repositório.

\begin{tabular}{|l|c|}
\hline \multicolumn{1}{|c|}{ Nome do repositório } & Quantidade de itens \\
\hline Open Research Data @PUC-Rio- & 31 \\
\hline GLOBE & 1.444 .964 \\
\hline Base de Dados Científicos da Universidade Federal do Paraná & 16 \\
\hline PPBio Data Repository & 1119 \\
\hline WorldClim & 412 \\
\hline IBICT Dataverse Network & 619 \\
\hline CEDAP Research Data Repository & 1108 \\
\hline
\end{tabular}

Fonte: Elaboração própria. 
Para possibilitar a visualização o repositório GLOBE foi excluído do gráfico a seguir, pois sua inclusão impossibilita a visualização da comparação, devido ao seu discrepante número de itens. Podemos perceber que existe uma grande diferença entre o número de itens de cada repositório, mesmo após desconsiderar o repositório GLOBE, com o PPBio apresentando o segundo maior número e a Base de Dados Científicos da Universidade Federal do Paraná, com a menor ocorrência o menor. A média de itens seria, então, 515 itens.

Imagem 2 - Gráfico de quantidade de itens.

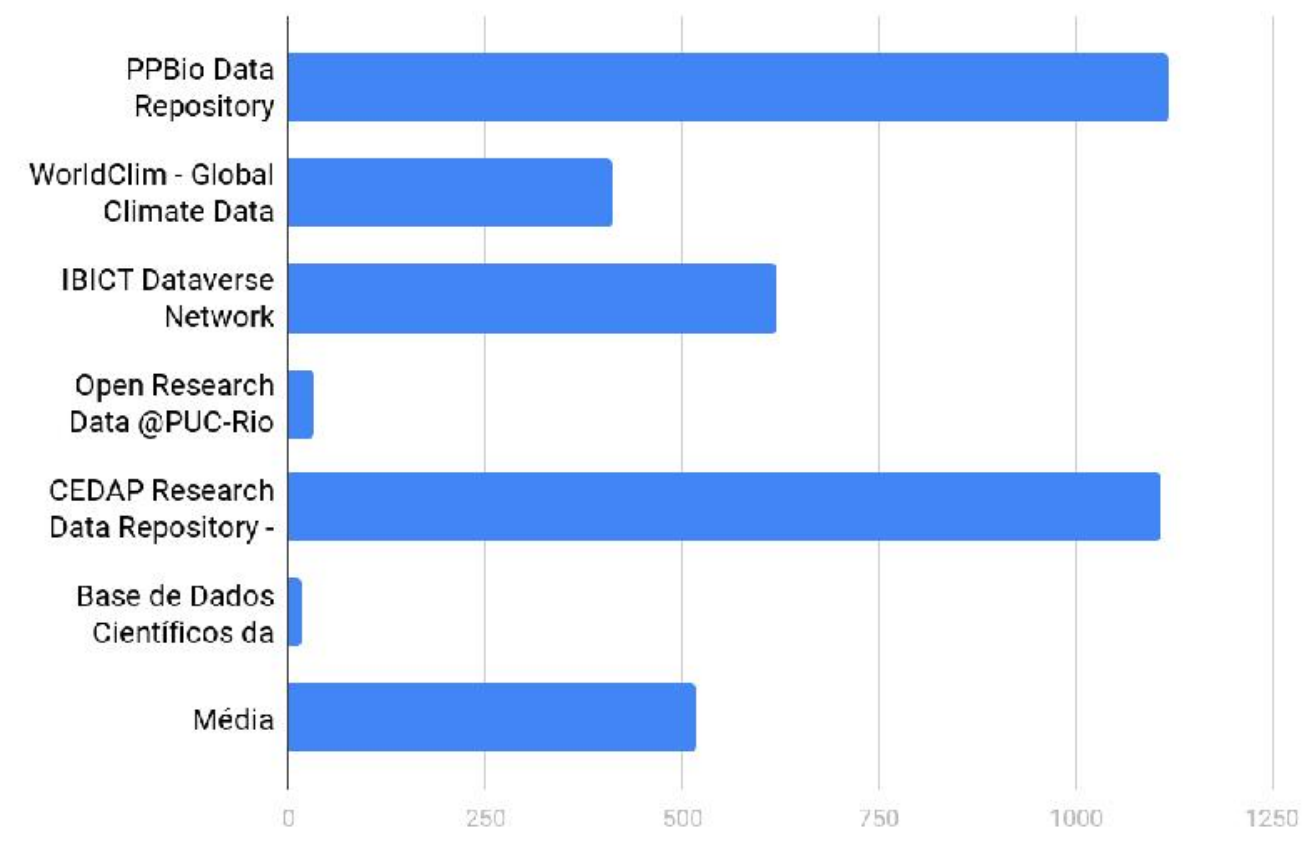

Fonte: Elaboração própria.

Os problemas de discrepância encontrados podem ter suas origens, exatamente, na própria definição do que é considerado um dado de pesquisa, que ainda não apresenta um consenso amplamente estabelecido.

\subsubsection{Relação do item com o conjunto e Relação do conjunto com a publicação}

As relações do item podem ser expressas em dois âmbitos, sua relação com o conjunto onde está inserido e sua relação com a publicação, a qual estaria associado. Foram, então, buscadas nos registros encontrados nos repositórios essas ocorrências, resultando na tabela a seguir: 


\begin{tabular}{|l|c|c|}
\hline \multicolumn{1}{|c|}{ Quadro 8 - Relações } \\
\hline Exploration and Production Data Bank & $\begin{array}{c}\text { Item - } \\
\text { Conjunto }\end{array}$ & $\begin{array}{c}\text { Conjunto - } \\
\text { Publicação }\end{array}$ \\
\hline Base de Dados Científicos da Universidade Federal do Paraná & Sim & Sim \\
\hline PPBio Data Repository & Sim & Não \\
\hline WorldClim & Não & Não \\
\hline GLOBE & Não & Não \\
\hline IBICT Dataverse Network & Sim & Sim \\
\hline International Ocean Discovery Program & Não & Não \\
\hline Open Research Data @PUC & Sim & Não \\
\hline CEDAP Research Data Repository & Sim & Não \\
\hline
\end{tabular}

Fonte: Elaboração própria.

Podemos perceber que cinco, dos nove repositórios, denotam a relação do item com o conjunto, enquanto apenas dois o fazem com a publicação. Totalizando, então, em apenas dois repositórios, que apresentam de forma completa as relações dos itens, enquanto quatro não mostraram relações de forma alguma.

\section{CONCLUSÕES}

As demandas atuais sobre dados de pesquisa e seu compartilhamento, que advêm da Ciência Aberta, apontam os repositórios de dados, como ferramentas para o armazenamento, organização, compartilhamento e divulgação desses dados. Porém, o baixo número de repositórios, a não padronização e a inconformidade com padrões internacionais estabelecidos são indicativos de que o Brasil tem muito trabalho a ser feito para poder se inserir nas contemporâneas práticas de Ciência Aberta. Outra característica que evidencia a situação é o fato de nenhum dos repositórios analisados possui certificações e apenas metade utiliza identificadores persistentes.

Não há dúvidas de que é necessário realizar um grande trabalho de conscientização da importância da Ciência Aberta para que o País possa contribuir para o avanço célere da Ciência. 


\section{REFERÊNCIAS}

COSTA, Michelli Pereira da; LEITE, Fernando César Lima. Repositórios institucionais de acesso aberto à informação científica: proposta de modelo de avaliação. RECIIS - RevEletron de Comun InfInov Saúde. Rio de Janeiro, v. 9, n. 3, 9 p. jul./set. 2015.

GIL, Antonio Carlos. Métodos e técnicas de pesquisa social. 6. ed. São Paulo : Atlas, 2008. RE3DATA, REGISTRY OF RESEARCH DATA REPOSITORIES. About. Disponível em: http://service.re3data.org/about. Acesso em: 22 de dez. de 2019.

RODRIGUES, Eloy; et. al. Os repositórios de dados científicos: estado da arte. 2010. 54 p. Disponível em: http://repositorium.sdum.uminho.pt/handle/1822/10830. Acesso em: 22 de dez. de 2019.

SARMENTO, Fernanda; et al. Algumas considerações sobre as principais declarações que suportam o movimento Acesso Livre. World Congresson Health Information and Libraries.

Salvador: 2005. Disponível em: <http://hdl.handle.net/10760/8512>. Acesso em: 22 de dez. de 2019. 